\title{
Evaluation of the Use of Postural Control Strategies during Dual-Tasks of Hearing-Impaired Athletes
}

\author{
Deniz Simsek', Caner Ozboke', Ela Arican Gultekin ${ }^{1}$

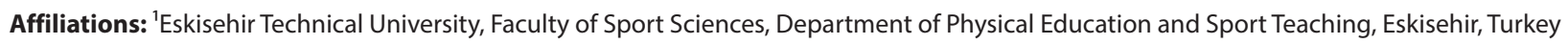

Correspondence: D. Simsek, Eskisehir Technical University, Faculty of Sport Sciences, Department of Physical Education and Sport Teaching, 2 Eylul Campus, Tepebasi, Eskisehir, Turkey. E-mail: deniz_yenigelen26@hotmail.com

\begin{abstract}
Dual-tasks are often used with postural control. These tasks, which generally target motor skills and cognitive performance, also help to determine the individual's postural control. The purpose of this study is to determine the changes in performance during the motor task, which includes the cognitive cues of the hearing-impaired athletes. A total of 31 hearing-impaired athletes ( $m a l e=19$, female $=12$ ) and 34 hearing-impaired sedentary people ( $m a l e=18$, female $=16$ ) were included voluntarily in the study. The FitLight Trainer ${ }^{\mathrm{TM}}$ system was used to determine participants' reaction time levels. The performance time of hearing-impaired male athletes was significantly lower than the hearing-impaired sedentary men in each of the three tests (Random Test: $t=4,089, p<0.05$; Cue Test: $t$ $=3,551, p<0.05$; Mixed Cue Test: $t=2,393, p<0.05)$. The performance time of hearing-impaired female athletes was statistically significantly lower than that of sedentary hearing-impaired females for all protocols (Random Test: $t=2,586, p<0,05$; Cue Test: $t=2,568, p<0.05$; Mixed Cue Test: $t=2,899, p<0.05)$. This study demonstrates that 1 ) hearing-impaired athletes perform postural control adjustments automatically during the motor task, and they require minimal less cognitive effort than they need to be minimally considered; 2 ) regular physical activities and training showed a positive development on other systems, especially the proprioceptive system, which controls balance. In future studies, dual-task reaction time values and postural control strategy comparisons should be measured among hearing-impaired athletes and athletes who do not have a hearing disability.
\end{abstract}

Keywords: postural control, motor skill, hearing-impaired, cognitive performance, dual-task

$@$ MJSSMontenegro

POSTURAL CONTROL STRATEGIES OF HEARING-IMPAIRED ATHLETES

http://mjssm.me/?sekcija=article\&artid=207

Cite this article: Simsek, D., Ozboke, C., \& Arican Gultekin, E. (2021). Evaluation of the Use of Postural Control Strategies during Dual-Tasks of Hearing-Impaired Athletes. Montenegrin Journal of Sports Science and Medicine, 10 (1), 11-17. doi: 10.26773/mjssm.210302

\section{Introduction}

Postural control can be defined as the ability of an individual to maintain a stable posture and remain standing when problems are encountered due to environmental conditions (Gallahue, Ozmun, \& Goodway, 2013). Adequate postural control requires the errorless operation of sensory systems and successful integration and regulation (Riemann \& Guskiew- $c z, 2000)$. Cooperation and the integration of sensory inputs, such as visual, vestibular, and proprioceptive, are essential to ensure postural stability (de Sousa, de França Barros, \& de Sousa Neto, 2012). Changes in sensory systems can also affect the structure of postural control, and postural control can be seen in a non-coordinated and impaired form (Plata, 1997). Since the vestibular system is essential in regulating postural 
control, hearing loss, vestibular system problems may affect postural control (Agmon, Lavie, \& Doumas, 2017). Dual-task testing is used to test deficits in postural control; such testing requires the simultaneous performance of the motor and cognitive tasks, such as standing, leaning, and taking a step. An individual's attentional resources and data processing capacity is probably limited, and they should be distributed among other tasks (Kerr, 1982). Recent studies suggesting that postural control requires a significant amount of attention have been working on maintaining an upright position using primary postural control source and dual-task testing (Woollacott \& Shumway-Cook, 2002; de Graaf-Peters, Blauw-Hospers, Dirks, Bakker, Bos, \& Hadders-Algra, 2007). In sports in which secondary performance is more important for winning, postural balance reduces the amount of attention and allows the body to focus on mental and/or motor skills. Most physical activities require athletes to provide motor responses to visual information by maintaining postural control. In this sense, studying the high demand for postural mechanisms in sports might shed light on unclear postural control strategies in both body stability and simultaneous mental or motor tasks.

In contrast, data on postural control skills in hearing-impaired individuals who participated in physical activities for an extended period, or any particular sports branch, is limited. This study aimed to determine the performance time deficits of hearing-impaired athletes during cognitive-motor dual tasks. Also, since postural control interacts with other tasks simultaneously (Derlich, Kręcisz, \& Kuczyński, 2011), dual-task testing is necessary to test postural control in individuals with hearing impairment. The determination of postural control of individuals with hearing-impaired via dual-task testing can be conducive to 1) a better understanding of differences between hearing-impaired athlete and sedentary individuals, 2) identifying possible effects of regular physical activity on postural control of hearing-impaired individuals, 3) the availability of a dual-task postural control protocol in hearing-impaired individuals.

\section{Methods}

\section{Participants}

A total of 65 individuals ((athletes; male $(\mathrm{n}=19)$ : age $=$ $21.3 \pm 2.01$ years, height $=176.73 \pm 7.84 \mathrm{~cm}$, weight $=74.8 \pm 6.4$ $\mathrm{kg}$; female $(\mathrm{n}=12)$ : age $=22.2 \pm 2.1$ years, height $=165.4 \pm 3.1$ $\mathrm{cm}$, weight $=63.7 \pm 8.0 \mathrm{~kg}$; Experience in Sports (year) $=9$ years; Total of Active Hours/Week (12 hours; 4 days a week, 3 hours per day)), and ((sedentary individuals; male $(\mathrm{n}=18)$ : age $=22.0 \pm 197$ years, height $=171.3 \pm 5.6 \mathrm{~cm}$, weight $=73.6 \pm 9.0$ $\mathrm{kg}$; female $(\mathrm{n}=16)$; age $=21.6 \pm 1.6$ years, height $=160.0 \pm 5.4 \mathrm{~cm}$, weight $=56.3 \pm 7.9 \mathrm{~kg})$ ) with degrees of hearing loss between 45 and $51 \mathrm{~dB}$ voluntarily participated in the study. Addiction, not using walking aids, not having major pain that restricts daily functions, and not having any known health problems are some of the criteria for the individuals to participate in the study. Both groups were matched according to their sexes and level of activeness (athlete/sedentary).

\section{Ethics}

The research protocol was approved by the university's Ethics Committee (Protocol No: 43180). To be able to include in this research, participants were asked to sign a consent form by the ethics committee's approval procedure.

\section{Data Collection}

In the research, to determine participants' reaction times, the FitLight Trainer system was used. It is a wireless reaction system with eight LED lights controlled by a tablet. The sensors can be deactivated by touching the lights as well as by merely hovering over the light. Also, the system allows the lights to be configured and the reaction time of the controller to be recorded. During the test, participants were asked to touch and deactivate the light as fast as possible.

\section{Tasks}

As in the study conducted by Laessoe, Grarup, and Bangshaab (2016), by placing eight lights 1.5 metres apart in three different colours at three different locations, participants were made to push their stability limits while attempting to reach and disable the lights. Four of the lights were placed in a blue zone on a wall in front of the participants. Two of the lights were placed in the red zone to the left of the participant, and the other two lights were placed in the green zone to the right of the participant. The pairs of lights were placed at shoulder and waist height, respectively. The lights in the red and the green zone were installed 3 metres apart from each other and 0.5 metres from the wall so that the participants would not block the lights while they were standing in the middle of the setup. The participants were asked to wait in the middle of the setup. To reach the lights, participants need to change their stability limits, or participants need to move their entire body to by taking a step. (Participants were expected to perform a similar motor task with a cognitive task in three trials throughout the dual-task test. However, the use of cognitive resources varies between tasks.) In each trial, the motor task consisted of 25 repetitive reaching tasks in which the participants were to hold their hands in front of one out of eight lights/sensors to turn off the light. The lights were adjusted to go off once, and once they are deactivated, the next one would go off in $0.5 \mathrm{sec}-$ onds. The number of activated lights in each different zone was evenly divided between each test to ensure the participants challenge their stabilities within and beyond their limits. In this manner, in all three tests, balance and postural control were challenged equally.

In the research, all three tests were different from each other in terms of cognitive demands. Participants were encouraged to use cognitive strategies with different possibilities by providing them cues about each task:

(1) The lights would be lit in random order (red, green, and blue). No cue was given as to where the next light would appear. In a cognitive sense, this test is expected to measure the reaction time of the participants.

(2) The colour of the light indicated the position of the next light. The purpose of this is to determine whether the participant was aware of the dual-tasks. If the light were red, the following light would be lit in the red zone. If the light were green, the following light would be lit in the green zone. If the light were blue, the following light would be in the blue zone.

(3) The colour of the light determines the position of the next light; however, the red and green cues were reversed. In other words, if the light were red, the following light would be in the green zone, and vice versa. The blue light, in contrast, indicates the next light would be in the blue zone. This task shows taking on cognitive skills in utilizing the given cues. The programmed order of the lights is shown in Figure 1. 


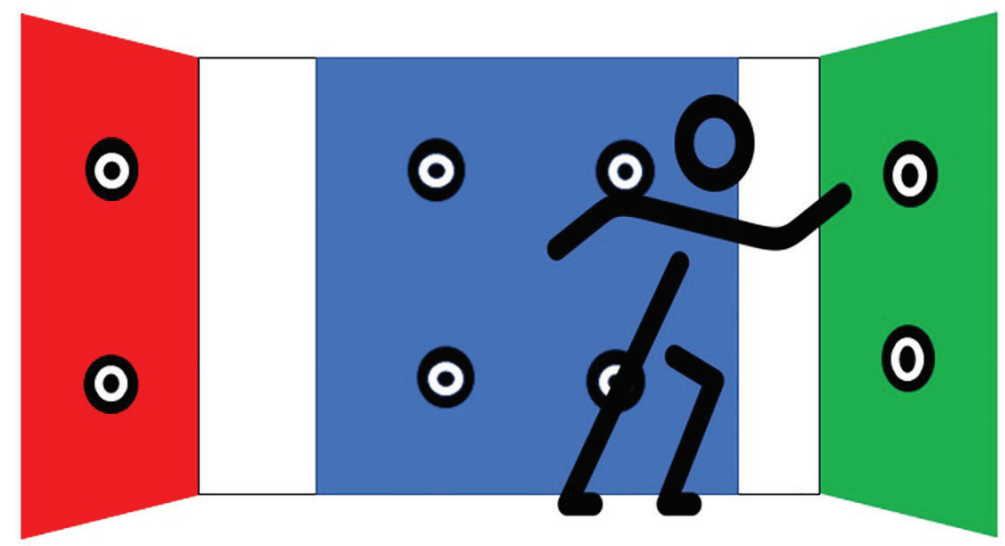

Random

\begin{tabular}{ccccccccccccccccccccccccccccc}
\hline $\begin{array}{c}\text { Light } \\
\text { No }\end{array}$ & 8 & 2 & 3 & 4 & 1 & 7 & 2 & 4 & 8 & 7 & 8 & 5 & 2 & 1 & 7 & 3 & 1 & 6 & 4 & 6 & 5 & 7 & 2 & 8 & 1 \\
Color & G & R & G & R & B & B & R & G & G & B & R & B & R & B & G & R & G & B & R & G & K & B & G & R & B \\
\hline $\begin{array}{c}\text { Light } \\
\text { No }\end{array}$ & 4 & 1 & 7 & 2 & 3 & 2 & 8 & 5 & 7 & 1 & 4 & 8 & 2 & 6 & 1 & 7 & 3 & 2 & 8 & 4 & 1 & 7 & 5 & 8 & 6 \\
Color & R & G & R & B & R & G & B & G & R & B & G & R & B & R & G & B & R & G & B & R & G & B & G & B & B \\
\hline $\begin{array}{c}\text { Light } \\
\text { No }\end{array}$ & 4 & 1 & 7 & 2 & 3 & 2 & 8 & 5 & 7 & 1 & 4 & 8 & 2 & 6 & 1 & 7 & 3 & 2 & 8 & 4 & 1 & 7 & 5 & 8 & 6 \\
Color & G & R & G & B & G & R & B & R & G & B & R & G & B & G & R & B & G & R & B & G & R & B & R & B & B
\end{tabular}

FIGURE 1. Installation. G: green zone; B: blue zone; R: red zone.

The lights were placed in three zones. Zone 1 and Zone 3 were marked as red and green, in the given order. The middle zone was blue. The light sequence was different in each test, but all lights were represented equally. In Trials 2 and 3, the colour of the light indicated the position of the following light.

The tests start with a light at a random point. All three tests were conducted in the order mentioned in the previous section. Before each test, the participants were informed about the procedure. Also, they were instructed to maintain (keeping a safe balance) their posture while they were deactivating the lights as fast as possible. The entire procedure was performed in two sessions with a 10-minute break in between. For participants to become used to the test and reliability of the research, a test-retest method was used in the study. For the validity of the research, only the data acquired from the second session were used.

\section{Data analysis}

For each test, the performance time was recorded automatically by the FitLight software and displayed on the system's control device. The figures were drawn manually entered in Excel. The data were analysed by using SPSS 23.0 (SPSS 23.0, Chicago, IL, USA). (Average times for sessions and groups are presented, and relative percentage changes are calculated for each session.) For each group and session, an average of performance time was presented, and the relative percentage values of Test 1 (random), Test 2 , and Test 3 (with the cue and random cue) were calculated. The Kolmogorov-Smirnov test was used to determine if the data were distributed normally. Reliability was calculated by absolute differences between the sessions and the intraclass correlation coefficient (ICCs). The ICCs were calculated by using an absolute agreement with a two-way mixed model (ICC $3,1)$. These values were read according to Kappa values by Landis and Koch: $<0.00$ is poor, $0.00-0.20$ is slight, $0.21-0.40$ is fair, $0.41-0.60$ is moderate, $0.61-0.80$ is substantial, and $0.81-1.00$ is almost perfect (Landis \& Koch, 1977). To refrain from any bias opinion regarding the participants' learning capacity, and for the validity of the research, only the data collected from the second session were used. To measure the performances in all three tests, factorial repeated ANOVA was used. Scores and deficits between the tests were shown as average and confidence intervals in the charts (CI 95\%). In the tests with given cues, performance improvements were evaluated according to the changes in the individual base scores. In the scope of this research, the significance level was accepted as $<.05$.

\section{Results}

The total and the average performance time of hearing-impaired athletes and sedentary individuals in three tests and their performance improvements in the cue given test were respectively shown in Table 1 (for female groups) and Table 3 (for male groups). The differences in average performance time between the two sessions in all three tests within the female and male groups were shown in Table 2 and Table 4, respectively. The hearing-impaired athletes and sedentary individuals' performance durations were better, as shown in Figure 2 . The relative improvement in performance times are significantly different $(\mathrm{p}<0.001)$, and during the cue given tests, this difference was smaller in sedentary individuals. Sedentary individuals' performance was mostly lower than the athletes' in all tests; however, both groups' performance improved (shorter amount of time) during the cue given motor tasks $(\mathrm{p}<0.001)$. Generally, sedentary groups' tests took significantly longer than athlete groups' tests did $(\mathrm{p}<0.001)$. When the baseline values were compared, there were improvements in the cue given tests. Improvement in the performance duration was more evident in simple cognitive tasks compared to cross-colour cue cognitive tasks. 
Table 1. Performances of Female Groups in Three Tests

\begin{tabular}{|c|c|c|c|c|c|c|}
\hline & Random & Cue & Mixed Cue & & Random vs Cue & Random vs Mixed Cue \\
\hline \multicolumn{7}{|c|}{ Sedentary Female } \\
\hline $1^{\text {st }}$ Session & $40.72(4.0)$ & $34.44(5.4)$ & $37.63(5.0)$ & & $-15.43 \% *$ & $-7.61 \%^{*}$ \\
\hline \multirow[t]{2}{*}{$2^{\text {nd }}$ Session } & $37.25(3.2)$ & $32.13(4.3)$ & $35.44(5.2)$ & & $-13.75 \% *$ & $-4.87 \%$ \\
\hline & & & & ICC & $0.67(0.22-0.88)$ & $0.79(0.52-0.92)$ \\
\hline \multicolumn{7}{|c|}{ Athletes Female } \\
\hline $1^{\text {st }}$ Session & $35.67(1.6)$ & $31.79(4.7)$ & $33.17(3.7)$ & & $-10.87 \% *$ & $-7.01 \%$ \\
\hline \multirow[t]{2}{*}{$2^{\text {nd }}$ Session } & $34.33(2.6)$ & $28.18(3.7)$ & $30.45(3.3)$ & & $-16.84 \% *$ & $-9.72 \%^{*}$ \\
\hline & & & & ICC & $0.59(0.10-0.87)$ & $0.56(0.10-0.84)$ \\
\hline
\end{tabular}

Note: ${ }^{*} p<0.05$. Total and average performance durations of female groups in three tests, and their performance improvements in the cue given tests. Time duration in seconds; average and standard deviation, increase in spent time, and average percentage (standard deviation). Negative values show performance improvements.

Time score in seconds; mean and SD, and reduction in performance improvements. ICC: Intra-class correlation cotime score, mean percentage (SD). Negative values represent efficient (CI 95\%).

Table 2. Value differences in Performance Durations of Female Groups in Tests

\begin{tabular}{|c|c|c|c|c|c|c|}
\hline & Protocols & Group & $\mathrm{n}$ & & Mean Differences & $\mathrm{P}$ \\
\hline \multirow{6}{*}{$1^{\text {st }}$ Session } & \multirow{2}{*}{ Random } & Sedentary Female & 16 & 40.72 & 5,052 & \multirow{2}{*}{, $000^{*}$} \\
\hline & & Athletes Female & 11 & 35.67 & $-5,052$ & \\
\hline & \multirow{2}{*}{ Cue } & Sedentary Female & 16 & 34.44 & 2,643 & \multirow{2}{*}{ 197 } \\
\hline & & Athletes Female & 11 & 31.79 & $-2,643$ & \\
\hline & \multirow{2}{*}{ Mixed Cue } & Sedentary Female & 16 & 37.63 & 4,452 & \multirow{2}{*}{, $018^{*}$} \\
\hline & & Athletes Female & 11 & 33.17 & $-4,452$ & \\
\hline \multirow{6}{*}{$2^{\text {nd }}$ Session } & \multirow{2}{*}{ Random } & Sedentary Female & 16 & 37.25 & 2.924 & \multirow{2}{*}{, $016^{*}$} \\
\hline & & Athletes Female & 11 & 34.33 & -2.924 & \\
\hline & \multirow{2}{*}{ Cue } & Sedentary Female & 16 & 32.13 & 3,955 & \multirow{2}{*}{, $016^{*}$} \\
\hline & & Athletes Female & 11 & 28.18 & $-3,955$ & \\
\hline & \multirow[t]{2}{*}{ Mixed Cue } & Sedentary Female & 16 & 35.44 & 4,987 & \multirow[t]{2}{*}{, $008^{*}$} \\
\hline & & Athletes Female & 11 & 30.45 & $-4,987$ & \\
\hline
\end{tabular}

Note: ${ }^{*} p<0.05$ Value differences in performance durations and their average in Session 1 and Session 2 of the female groups in all three tests. Relative improvements in performances in cue given tests are higher in hearing-impaired female athletes compared to hearing-impaired sedentary female participants $(p<0.001)$. Also, performance duration time is longer in hearing-impaired sedentary female participants in cross-location cue test $(p<0.01$; Figure 3$)$.

Relative improvements in performances in cue given tests are higher in hearing-impaired female athletes compared to hearing-impaired sedentary female participants $(\mathrm{p}<0.001)$.
Also, performance time is shorter in hearing-impaired female athletes in the cross-location cue test $(\mathrm{p}<0.01)$.

Hearing-impaired sedentary participants were generally
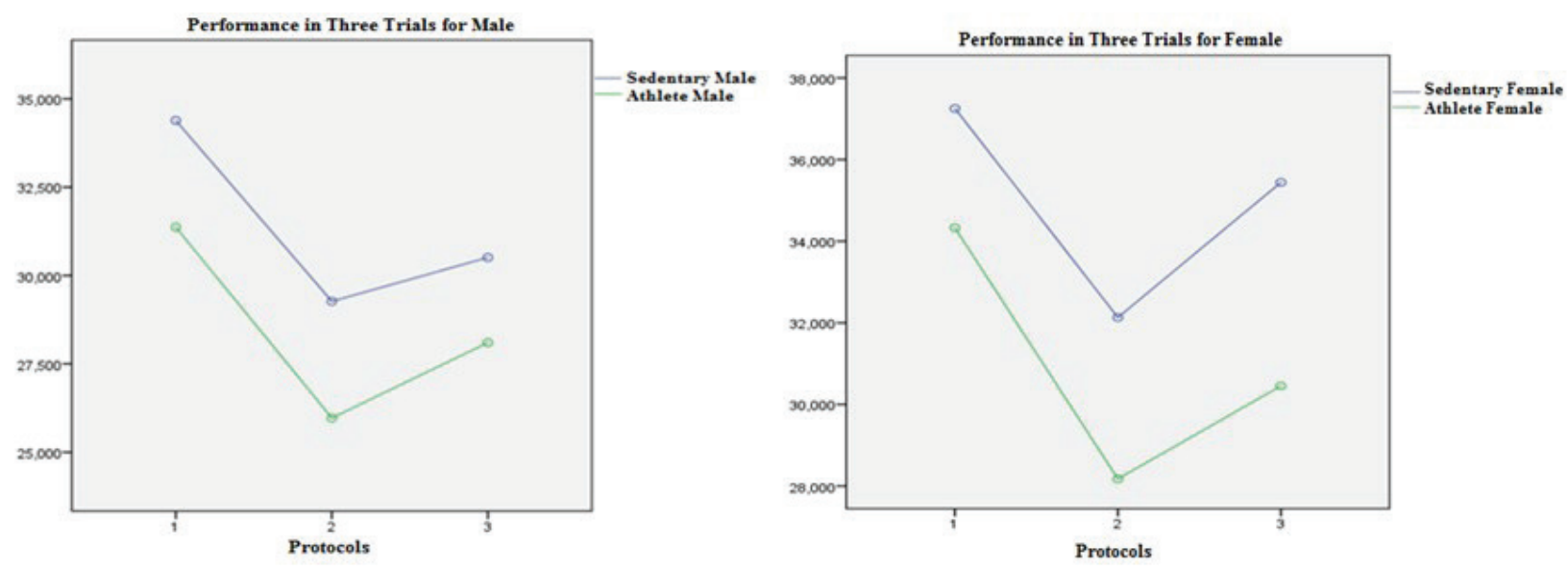

FIGURE 2. Performance Duration

slower than the hearing-impaired athletes in all trials, but both groups (sedentary and athletes) improved their performance (i.e., used shorter time) when they were provided with a leading cue $(\mathrm{p}<0.001)$. 
Table 3. Performances of Male Groups in Three Tests

\begin{tabular}{rccccc}
\hline & Random & Cue & Mixed Cue & Random vs. Cue & Random vs. Mixed Cue \\
\hline \multirow{3}{*}{$1^{\text {st }}$ Session } & $37.53(2.4)$ & Sedentary Male & & \\
$2^{\text {nd }}$ Session & $34.39(2.2)$ & $29.27(3.2)$ & $30.51(3.4)$ & $-14.89 \%^{*}$ & $-11.42 \%^{*}$ \\
& & & ICC & $0.51(0.07-0.79)$ \\
\hline & & Athletes Male & & $-11.27 \%^{*}$ \\
$1^{\text {st }}$ Session & $32.89(3.0)$ & $28.08(1.8)$ & $30.37(3.0)$ & $-14.62 \%^{*}$ & $-7.67 \%^{*}$ \\
$2^{\text {nd }}$ Session & $31.37(2.3)$ & $25.97(2.4)$ & $28.10(2.7)$ & $-17.22 \%^{*}$ & $-10.42 \%^{*}$ \\
& & & ICC & $0.57(0.08-0.83)$ \\
\hline
\end{tabular}

Note. ${ }^{*} p<0.05$. Total and average performance durations of male groups in three tests, and performance improvements in the cue given tests. Time duration in seconds; average and standard deviation, increase in spent time, and average percentage (standard deviation). Negative values show performance improvements.

Time score in seconds; mean and SD, and reduction in time score, mean percentage (SD). Negative values represent

performance improvements. ICC: Intra-class correlation coefficient (CI 95\%).

Table 4. Value differences in Performance Durations of Male Groups in Tests

\begin{tabular}{|c|c|c|c|c|c|c|}
\hline & Protocols & Group & $\mathrm{n}$ & & Mean Differences & $P$ \\
\hline \multirow{6}{*}{$1^{\text {st }}$ Session } & \multirow[t]{2}{*}{ Random } & Sedentary Male & 18 & 37.53 & 4.638 & $0.000^{*}$ \\
\hline & & Male Athletes & 19 & 32.89 & -4.638 & \\
\hline & \multirow[t]{2}{*}{ Cue } & Sedentary Male & 18 & 33.44 & 5.358 & $0.000^{*}$ \\
\hline & & Male Athletes & 19 & 28.08 & -5.358 & \\
\hline & \multirow[t]{2}{*}{ Mixed Cue } & Sedentary Male & 18 & 33.24 & 2.874 & $0.007^{*}$ \\
\hline & & Male Athletes & 19 & 30.37 & -2.874 & \\
\hline \multirow{6}{*}{$2^{\text {nd }}$ Session } & \multirow[t]{2}{*}{ Random } & Sedentary Male & 18 & 34.39 & 3,019 & $0.000^{*}$ \\
\hline & & Male Athletes & 19 & 31.37 & $-3,019$ & \\
\hline & \multirow[t]{2}{*}{ Cue } & Sedentary Male & 18 & 29.27 & 3,302 & $0.001^{*}$ \\
\hline & & Male Athletes & 19 & 25.97 & $-3,302$ & \\
\hline & \multirow[t]{2}{*}{ Mixed Cue } & Sedentary Male & 18 & 30.51 & 2,411 & $0.022^{*}$ \\
\hline & & Male Athletes & 19 & 28.10 & $-2,411$ & \\
\hline
\end{tabular}

Note. ${ }^{*} p<0.05$. Value differences of performance durations and their average in Session 1 and Session 2 of the male groups in all three tests. Relative improvements in performances in cue given tests are higher in hearing-impaired male athletes compared to hearing-impaired sedentary female participants $(p<0.001)$. Further, performance duration time is longer in hearing-impaired sedentary male participants in cross-location cue test $(p<0.01$; Figure 5).

Relative improvements in performances in cue given tests are higher in hearing-impaired male athletes compared to hearing-impaired sedentary male participants $(p<0.001)$. Furthermore, performance time is shorter in hearing-impaired male athletes in the cross-location cue test $(\mathrm{p}<0.01)$.

\section{Discussion}

The purpose of the conducted research is to determine the changes in performance durations during motor tasks that include cognitive cues of hearing-impaired athletes. As hypothesized, the results suggest that hearing-impaired female and male athletes had better performance compared to hearing-impaired sedentary individuals in the tests with or without cues given. Furthermore, the results show that hearing-impaired sedentary individuals do not use the information in the cues. There are several possible explanations for this result. A possible satisfactory explanation for the hearing-impaired athletes' minimal decrease in the second task performance during the dual-tasks is that athletes use cognitive resources less. Considering sedentary individuals' guessing the location of the next light and using expected postural control strategies, the increase in the reaction time supports the idea of shifting focus from posture to cognition. This may be an indication of automatic postural control requiring more activity of the central nervous system, which can be explained with Abernethy's model, which shows the attentional capacity sharing hypothesis (Abernethy, 1988).

According to that model, when the primary task is more complicated, a greater portion of an individual's processing capacity needs to be shared to maintain a sustainable performance (Huang \& Mercer, 2001). In our study, according to this model, since the hearing-impaired sedentary group could not use the given cues as much as the hearing-impaired athletes could, the sustained attentional capacity of the sedentary individuals might be insufficient in this research. Also, this can be explained as a result of motor tasks requiring more attention, or attentional capacity of sedentary individuals' being limited. Because exercising is simultaneously performed with not only a motor but also a cognitive task in a distracting environment, it can be pointed out that hearing-impaired athletes' motor learning process can affect the results. Also, several studies suggest that maintaining postural control is an indication of an autonomous skill level (Stins, Michielsen, Roerdink, \& Beek, 2009; Donker, Roerdink, Greven, \& Beek, 2007). This 
finding may be responsible for that fact that hearing-impaired individuals' with nine years of experience in sports show that exercising has an impact on disabled individuals' developing postural control. This also demonstrates the reason that both female and male hearing-impaired athletes performed better during the tests compared to sedentary hearing-impaired individuals since that cognitive demand is minimal for the athlete groups.

These findings generally concurred with those of previous studies; that investigated individuals with different groups of people (Howell, Beasley, Vopat, \& Meehan III, 2017; Martini, Goulet, Gates, \& Broglio, 2016; Howell, Osternig, \& Chou, 2015). While most of the studies (Cossette, Ouellet, \& McFadyen, 2014; Howell, Osternig, Koester, \& Chou, 2014) are evaluated based on cognitive performance reaction time, some researchers studied walking variables and changes in the reaction time (Cossette et al., 2014). Another critical indicator used to improve stability and cognitive performance is physical exercise (Zanotto et al., 2014; Gomez-Pinilla \& Hillman, 2013). Our results are consistent with the referenced results (Brown \& Bennett, 2002; Park \& Reuter-Lorenz, 2009; Wollesen \& Voelcker-Rehage, 2014). It has been indicated that training has great importance in improving various cognitive skills and reducing cognitive-motor intervention (Brown \& Bennett, 2002; Park \& Reuter-Lorenz, 2009; Wollesen \& Voelcker-Rehage, 2014). At the end of their research, Müller and Blischke (2009) proposed that dual-task costs are reduced by training, which allows modulation of consciousness-dependent motor activities to be more automatic. As proposed by Smith and Chambertlin (1992), soccer players ran slowly with the addition of the secondary tasks; however, they also determined that players' speed increased as the years of experience increased. In their studies, Vuillerme and Nougier (2004) have that while conducting more complicated balance tasks, expert gymnasts use less cognitive interference compared to beginners.

These results show that during testing and learning a new skill, the task components are more focused on, and this might not be the case for expert athletes who have higher skill levels. This study demonstrates that athletes who display better performance in secondary tasks can train in more complicated situations where they have to guess their opponent's moves. In contrast, it is thought that athletes who display worse performance in the secondary tasks might need more practice on basic skills during single tasks or less complicated dual-tasks.

There are a few limitations to this research. First, the number of participants can be increased. The reliability of the data will increase when there are more participants included in the research. Second, in future studies, dual-task reaction time values and postural control strategy comparisons should be measured among hearing-impaired athletes and athletes who do not have a hearing disability.

\section{References}

Abernethy, B. (1988). Dual-task methodology and motor skills research: some applications and methodological constraints. Journal of Human Movement Studies, 14, 101 132.

Agmon, M., Lavie, L., \& Doumas, M. (2017). The association between hearing loss, postural control, and mobility in older adults: A systematic review. Journal of the American Academy of Audiology, 28(6), 575-588. doi: 10.3766/ jaaa. 16044

Brown, S., \& Bennett, E. (2002). The role of practice and automaticity in temporal and nontemporal dual-task performance. Psychological Research, 66(1), 80-89. doi: $10.1007 / \mathrm{s} 004260100076$

Cossette, I., Ouellet, M. C., \& McFadyen, B. J. (2014). A preliminary study to identify locomotor-cognitive dual tasks that reveal persistent executive dysfunction after mild traumatic brain injury. Archives of physical medicine and rehabilitation, 95(8), 1594-1597. doi: 10.1016/j. apmr.2014.03.019

de Graaf-Peters, V. B., Blauw-Hospers, C. H., Dirks, T., Bakker, H., Bos, A. F., \& Hadders-Algra, M. (2007). Development of postural control in typically developing children and children with cerebral palsy: possibilities for intervention? Neuroscience \& Biobehavioral Reviews, 31(8), 1191-1200. doi: 10.1016/j.neubiorev.2007.04.008

de Sousa, A. M. M., de França Barros, J., \& de Sousa Neto, B. M. (2012). Postural control in children with typical development and children with profound hearing loss. International journal of general medicine, 5, 433. doi: 10.2147/IJGM.S28693

Derlich, M., Kręcisz, K., \& Kuczyński, M. (2011). Attention demand and postural control in children with a hearing deficit. Research in developmental disabilities, 32(5), 18081813. doi: 10.1016/j.ridd.2011.03.009

Donker, S. F., Roerdink, M., Greven, A. J., \& Beek, P. J. (2007). The regularity of center-of-pressure trajectories depends on the amount of attention invested in postural control. Experimental Brain Research, 181, 1-11. doi: 10.1007/ s00221-007-0905-4

Gallahue, D.L., Ozmun J.C. ve Goodway J.D. (2013). Understanding motor development: Infants, children, adolescents, adults. New York: McGraw-Hill.

Gomez-Pinilla, F., \& Hillman, C. (2013). The influence of exercise on cognitive abilities. Comprehensive Physiology, 3(1), 403-428. doi: 10.1002/cphy.c110063

Howell, D. R., Beasley, M., Vopat, L., \& Meehan III, W. P. (2017). The effect of prior concussion history on dual-task gait following a concussion. Journal of neurotrauma, 34(4), 838-844. doi: 10.1089/neu.2016.4609

Howell, D. R., Osternig, L. R., \& Chou, L. S. (2015). Adolescents demonstrate greater gait balance control deficits after a concussion than young adults. The American journal of sports medicine, 43(3), 625-632. doi: $10.1177 / 0363546514560994$

Howell, D. R., Osternig, L. R., Koester, M. C., \& Chou, L. S. (2014). The effect of cognitive task complexity on gait stability in adolescents following a concussion. Experimental brain research, 232(6), 1773-1782. doi: 10.1007/s00221-014-3869-1

Hsiao, E. T., \& Robinovitch, S. N. (1997). Common protective movements govern unexpected falls from standing height. Journal of biomechanics, 31(1), 1-9. doi: 10.1016/ S0021-9290(97)00114-0

Huang, H. J., \& Mercer, V. S. (2001). Dual-task methodology: applications in studies of cognitive and motor performance in adults and children. Pediatric Physical Therapy, 13(3), 133-140.

Kerr R. (1982). Psychomotor Learning. Philadelphia: Saunders College Pub.

Laessoe, U., Grarup, B., \& Bangshaab, J. (2016). The use of 
cognitive cues for anticipatory strategies in a dynamic postural control task-validation of a novel approach to dual-task testing. PloS one, 11(8). doi: 10.1371/journal. pone. 0157421

Landis, J. R., \& Koch, G. G. (1977). An application of hierarchical kappa-type statistics in the assessment of majority agreement among multiple observers. Biometrics, 363-374. doi: 10.2307/2529786

Martini, D. N., Goulet, G. C., Gates, D. H., \& Broglio, S. P. (2016). Long-term effects of adolescent concussion history on gait, across age. Gait \& posture, 49, 264-270. doi: 10.1016/j.gaitpost.2016.06.028

Müller, H., \& Blischke, K. (2009). Motorisches Lernen. Grundlagen der Sportpsychologie, 159-228.

Park, D. C., \& Reuter-Lorenz, P. (2009). The adaptive brain: aging and neurocognitive scaffolding. Annual review of psychology, 60, 173-196. doi: 10.1146/annurev. psych.59.103006.093656

Plata, AE. (1997). Understanding the roles of vision in the control of human locomotion. Gait Posture. 5, 54-69. doi: 10.1016/S0966-6362(96)01109-5

Riemann, B. L., \& Guskiewicz, K. M. (2000). Effects of mild head injury on postural stability as measured through clinical balance testing. Journal of athletic training, 35(1), 19.

Robinovitch, S. N., Hayes, W. C., \& McMahon, T. A. (1991) Prediction of femoral impact forces in falls on the hip. Journal of biomechanical engineering, 113(4), 366-374 doi: $10.1115 / 1.2895414$

Smith, M. D., \& Chambertlin, C. J. (1992). Effect of adding cognitively demanding tasks on soccer skill performance. Perceptual and Motor Skills, 75, 955-961. doi: 10.2466/ pms.1992.75.3.955

Stins, J. F., Michielsen, M. E., Roerdink, M., \& Beek, P. J. (2009). Sway regularity reflects attentional involvement in postural control: Effects of expertise, vision, and cognition. Gait \& Posture, 30(1), 106-109. doi: 10.1016/j. gaitpost.2009.04.001

Vuillerme, N., \& Nougier, V. (2004). Attentional demand for regulating postural sway: The effect of expertise in gymnastics. Brain Research Bulletin, 63(2), 161-165. doi: 10.1016/j.brainresbull.2004.02.006

Wollesen, B., \& Voelcker-Rehage, C. (2014). Training effects on motor-cognitive dual-task performance in older adults. European Review of Aging and Physical Activity, 11(1), 5. doi: 10.1007/s11556-013-0122-z

Woollacott, M., \& Shumway-Cook, A. (2002). Attention and the control of posture and gait: a review of an emerging area of research. Gait \& posture, 16(1), 1-14. doi: 10.1016/ S0966-6362(01)00156-4

Zanotto, T., Bergamin, M., Roman, F., C Sieverdes, J., Gobbo, S., Zaccaria, M., \& Ermolao, A. (2014). Effect of exercise on dual-task and balance on the elderly in multiple disease conditions. Current aging science, 7(2), 115-136. doi: 10.21 74/1874609807666140328095544 